\title{
Meta-prediction of MTHFR gene polymorphism-mutations, air pollution, and risks of leukemia among world populations
}

\author{
Shin-Yu A. Lien ${ }^{1,2}$, Lufei Young ${ }^{3}$, Bih-Shya Gau ${ }^{4}$, S. Pamela K. Shiao ${ }^{3}$ \\ ${ }^{1}$ School of Nursing, College of Medicine, Chang Gung University, Taoyuan, Taiwan (R.O.C.) \\ ${ }^{2}$ Division of Endocrinology and Metabolism, Department of Internal Medicine, Chang Gung Memorial Hospital Linkou Branch, \\ Taoyuan, Taiwan (R.O.C.) \\ ${ }^{3}$ College of Nursing, Augusta University, Augusta, Gerogia, USA \\ ${ }^{4}$ School of Nursing, College of Medicine, National Taiwan University, Taipei, Taiwan (R.O.C.) \\ Correspondence to: S. Pamela K. Shiao, email: pshiao@msn.com
}

Keywords: meta-analysis, meta-prediction, methylenetetrahydrofolate reductase, leukemia, air pollution

Received: April 15, 2016

Accepted: December 05, 2016

Published: December 10, 2016

\section{ABSTRACT}

The major objective of this study was to examine the association between Methylenetetrahydrofolate Reductase (MTHFR) polymorphisms and the risk of various types of leukemias across the lifespans of children and adults by using the metapredictive techniques. The secondary objective was to examine the interactions among epigenetic risk factors (including air pollution), MTHFR polymorphisms, and the risks of developing leukemia. We completed a comprehensive search of 6 databases to find 54 studies (10,033 leukemia cases and 15,835 controls) for MTHFR 677, and 43 studies (8,868 cases and 14,301 controls) for MTHFR 1298, published from 1999 to 2014. The results revealed that, in European populations; childhood populations; children from Europe, East Asia, and America; and children with acute lymphocytic leukemia (ALL), MTHFR 677 polymorphisms (both TT and CT types together and individually) are protective, while CC wildtype was leukemogenic. In addition, MTHFR 1298 polymorphisms were protective against ALL and acute myeloid leukemia in European children, and in chronic myeloid leukemia in all adults worldwide and American adults. Air pollution played a role in the increased polymorphisms of MTHFR 677 genotypes in childhood leukemia.

\section{INTRODUCTION}

Leukemia is one of the most common types of hematological malignancy among children [1] and adults worldwide [2, 3]. The etiology of leukemia remains mostly unknown; however, it is likely the accumulation of genetic and epigenetic alterations induced by adverse gene-environment interactions [4-6]. The role of methylenetetrahydrofolate reductase (MTHFR) polymorphisms in the development of leukemia has been extensively examined; however, the evidence about its role in leukemogenesis is inconsistent. The MTHFR is a key enzyme in folate metabolism and DNA methylation [7]. MTHFR enzyme converts homocysteine to methionine in the methylation pathways, which helps generation of 1 million new cells and DNA molecules as well as protein and lipid syntheses daily in humans $[8,9]$. Two common polymorphisms in MTHFR, C677T (rs 1801133) and A1298C (rs 1801131), have been associated with reduced MTHFR enzymatic activity, which leads to hyperhomocysteinemia, aberrant folate metabolism, and DNA hypomethylation [5]. Abnormal folate metabolism has been found to cause DNA translocations and deletions in hematopoietic progenitor cells, triggering the development of leukemia $[5,7,8]$.

However, other studies have reported a protective advantage of carrying MTHFR variant alleles against leukemia in both children and adults $[9,10]$. Leukemias, just like colorectal carcinomas, are derived from rapid proliferations of tissues, which requires faster DNA synthesis and folate metabolism [11]. Therefore, leukemogenesis would be reduced by DNA hypomethylation at the local tissues and reduced MTHFR enzymatic activities at the bone marrows where active leukemogenesis occurs in the carriers of MTHFR polymorphisms $[6,8,12]$. Further, evidence has shown 
that environmental factors and lifestyle behaviors have played pivotal roles in developing leukemia through modulating methylation pathways [13] and alternating folate metabolism [14-16]. For example, many studies have supported the association between childhood leukemia and exposure to air pollutants [17-23]. Outdoor air pollution containing substances such as nitrogen dioxide, benzene, and polycyclic aromatic hydrocarbons, has been classified as carcinogenic $[18,19]$. Benzene in particular has potent hematotoxicity and carcinogenicity. Besides linking direct exposure and cancer, studies also reported the increased leukemia risk among children whose mothers had occupational exposure to benzene $[13,17,24]$. Additional studies reported the potential leukemogenesis effect of benzene in children could occur even at ambient air levels lower than currently allowed limits [17, 18]. Despite these findings, previous metaanalyses did not examine the effects of gene-environment interactions, specifically air pollution, on the associations with MTHFR polymorphisms and leukemia risk [25-27]. To fill this gap in the evidence $[28,29]$, we conducted a meta-analysis with meta-predictive techniques to examine the impact of exposure to air pollution on the role of MTHFR polymorphism in leukemogenesis of various leukemias across lifespans of children and adults.

\section{RESULTS}

\section{Characteristics of the studies}

We have summarized how we selected studies in Figure 1. A total of 92 articles were initially identified between 1965 and August 2016. We located a total of 54 eligible studies (10,033 leukemia cases and 15,835 controls) for MTHFR 677 and 43 studies (8,868 leukemia cases and 14,301 controls) for MTHFR 1298 polymorphisms. These studies were conducted in five continents, included both childhood and adulthood leukemias, and specified subtypes: acute lymphoblastic leukemia (ALL), acute myeloid leukemia (AML), chronic myeloid leukemia (CML), and various combinations of mixed types (Supplementary Table S1, Supplementary Figure S1).

\section{Pooled analyses by leukemia types and age groups}

For all included study groups, the pooled analysis showed significant associations between MTHFR 677 TT and TT plus CT polymorphisms as protective against leukemogenesis in 16 European studies (See Table 1A, and Supplementary Table S2). In addition, for children, MTHFR 677 TT and CT polymorphisms together were protective against leukemia $(\mathrm{RR}=0.96,95 \%$ confidence interval $[\mathrm{CI}=0.93,0.99], p=.0154)$, while $\mathrm{CC}$ wildtype was associated with increased risk of leukemia $(\mathrm{RR}=1.04,95 \% \mathrm{CI}[1.01,1.08], p=.0139)$ (Table 1A). Subgroup analyses by regions showed that MTHFR 677 polymorphisms were protective against leukemia in children from Europe, East Asia, and America (Europe-CT type: $\mathrm{RR}=0.94,95 \% \mathrm{CI}[0.89,0.99], p=.0316$; $\mathrm{TT}+\mathrm{CT}$ types: $\mathrm{RR}=0.94,95 \% \mathrm{CI}[0.90,0.99], p=.0187$; East Asia-CT type: $\mathrm{RR}=0.93,95 \%$ CI $[0.86,1.00], p=.0491$; America - TT type: $\mathrm{RR}=0.70,95 \% \mathrm{CI}[0.52,0.95]$, $p=.0212$; TT $+\mathrm{CT}$ types: $\mathrm{RR}=0.83,95 \% \mathrm{CI}[0.68,0.99]$, $p=.0449$ ) (Table 1A, Table 2). On the other hand, MTHFR $677 \mathrm{CC}$ wildtype was a risk genotype for developing leukemia in children from Europe and America (Europe: $\mathrm{RR}=1.11,95 \% \mathrm{CI}[1.02,1.22], p=.0207$; America: $\mathrm{RR}=1.09,95 \%$ CI $[1.02,1.20], p=.0451)$. Subgroup analyses by leukemia types again demonstrated the protective role of MTHFR 677 TT polymorphism against ALL in American children $(\mathrm{RR}=0.71,95 \% \mathrm{CI}$ $[.51,0.99], p=.0405)$ while MTHFR $677 \mathrm{CC}$ and CT genotypes increased risk of ALL in American children $(\mathrm{RR}=1.05,95 \% \mathrm{CI}[1.00,1.09], p=.0388)$. The pooled meta-analyses did not show significant roles of MTHFR 677 polymorphisms in any of the adulthood leukemias (Table 1A, Table 2).

Similar to MTHFR 677, MTHFR 1298 CC and AC polymorphisms played protective roles against ALL and AML in European children, cases aggregated, in 4 studies $(\mathrm{RR}=0.89,95 \%$ CI $[.82, .98], p=.0124)$, while the AA wildtype was associated with increased risk of ALL and AML, cases aggregated, in the same studies $(\mathrm{RR}=1.12$, $95 \%$ CI [1.03, 1.23], $p=.0126$ ) (Table 1B, Supplementary Table S3). Similarly, in 7 studies conducted worldwide that included adults, MTHFR $1298 \mathrm{CC}$ and AC polymorphisms were protective against $\mathrm{CML}(\mathrm{RR}=0.82,95 \% \mathrm{CI}$ $[0.66,1.01], p=.0421)$ while AA wildtype was a risk type for developing CML (worldwide, 7 studies: $\mathrm{RR}=1.14,95 \% \mathrm{CI}[1.00,1.31], p=.0477$; America, 3 studies: $\mathrm{RR}=1.18,95 \%$ CI $[1.01,1.38], p=.0416$ ) (Table 1B). The results from both funnel plot and Egger's linear regression test showed no significant publication bias in any eligible studies.

\section{Subgroup analyses by countries}

To identify sources of heterogeneity, we further analyzed subgroups of countries, grouping them by risk for childhood leukemias (Table 2). We divided countries based on MTHFR 677 polymorphisms (TT plus CT together) being protective $(\mathrm{RR}<1)$, risks $(\mathrm{RR}>1)$, or mixed effects ( $\mathrm{RR}$ varied around 1). The countries that had MTHFR polymorphisms as protective genotypes $(\mathrm{RR}<1)$ included mostly European countries (Serbia, Slovenia, Greece, Netherlands, United Kingdom [UK], Portugal), plus Brazil and Singapore (Figure 2A). The countries that had MTHFR polymorphisms as risk genotypes $(\mathrm{RR}>1)$ included Canada, Taiwan, the Philippines, India, Iran and Egypt (Figure 2B). With mixed effects, MTHFR 677 polymorphisms had varied effects on development of childhood leukemias in Germany, South Korea, China, Indonesia and Turkey (Figure 2C). 
To validate the heterogeneous findings, we further utilized a geographic information system (GIS) to visualize regional distributions [30]. The global maps demonstrated the variations in the distribution of MTHFR 677 polymorphisms and their inconsistent roles in leukemogenesis in children across regions (Supplementary Figures S2, S3, and S4). In the first two GIS maps, we used the continuous color spectrum from yellow to red, representing the increasing levels of polymorphisms, and, in the third map, red-green colors - red indicating leukemia risk, and green indicating protective effects. Similar to the pooled meta-analysis, GIS maps showed that MTHFR 677 polymorphisms individually (CT, TT) and together (TT and CT) played a protective role against childhood leukemia in most countries except Taiwan, China, India, Turkey, and Egypt (Supplementary Figures S2, S3, S4).

\section{Meta-prediction}

Given the role of heterogeneous findings on the effects of MTHFR polymorphisms, we performed meta-predictive analyses using both big-data analytics and conventional analyses on childhood leukemias (Table 3). We used both partition tree and Turkey's tests to examine the potential interaction between air pollution and polymorphisms, and their combined impact on leukemia risk. Based on guidelines from the World Health Organization [32] on air quality measures, we used the death from air pollution (AP death) as the measure for air quality (Level 2 $=50-100$ or less annual deaths/ million population; Level $3=100-250$ deaths/million; and Level $4=250-400$ or greater deaths/million) [33]. The partition tree and Tukey's tests showed significant differences between groups exposed to low versus

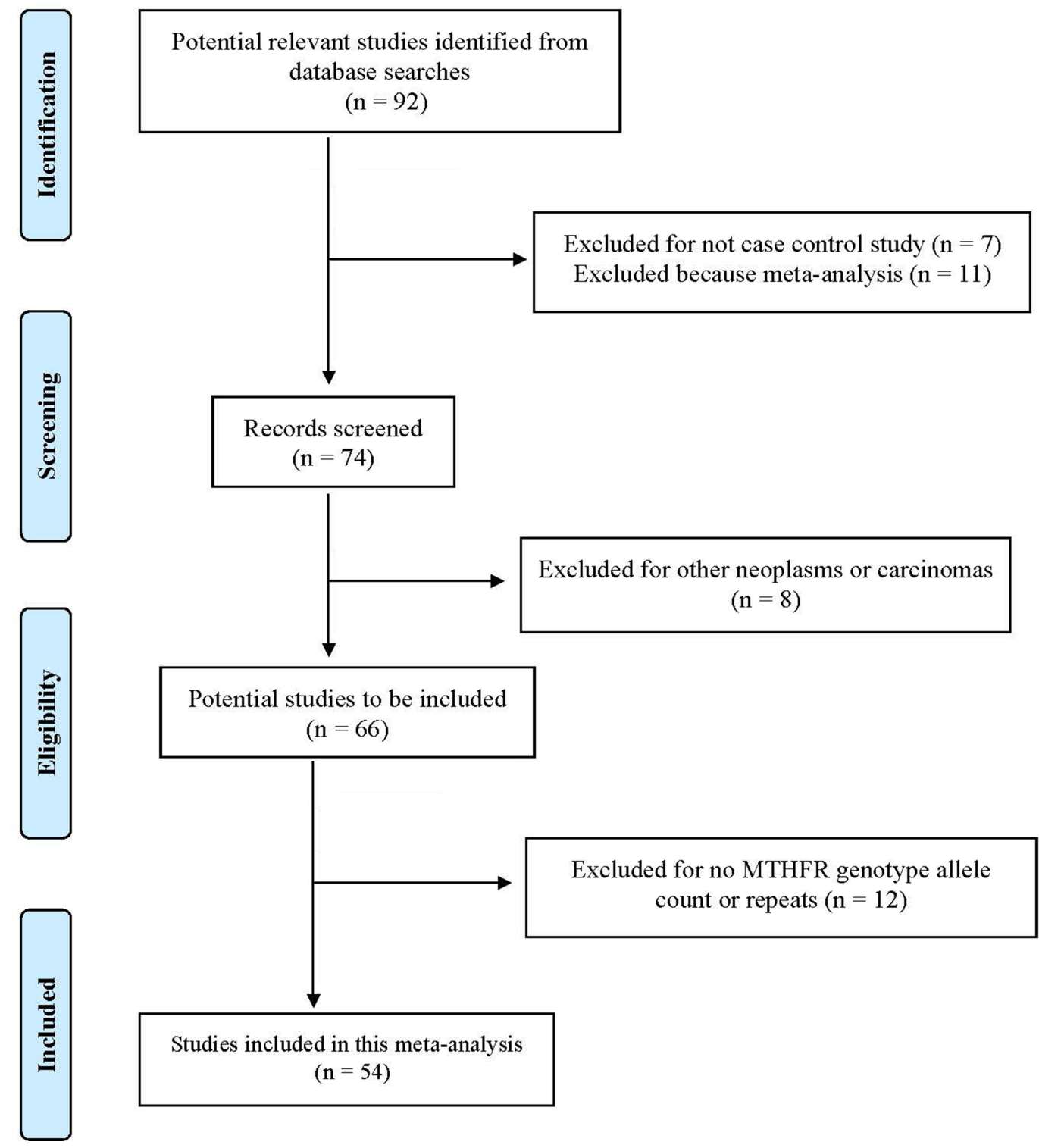

Figure 1: Progression by which studies were selected for this meta-analysis. 
Table 1A: Schema of significant findings across studies on MTHFR 677 genotypes and risk of leukemia

\begin{tabular}{|c|c|c|c|c|c|c|c|c|c|}
\hline NTHFR 677 & 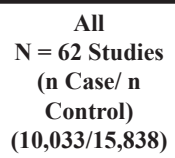 & $\begin{array}{l}\text { All children } \\
\text { 39 Studies } \\
(5,614 / 8,474)\end{array}$ & $\begin{array}{c}\text { ALL } \\
\text { 35 Studies } \\
(5,161 / 7,796)\end{array}$ & $\begin{array}{l}\text { ALL+AML } \\
\text { 4 Studies } \\
(1,331 / 1,304)\end{array}$ & $\begin{array}{c}\text { All adults } \\
23 \text { Studies } \\
(3,554 / 6,575)\end{array}$ & $\begin{array}{c}\text { ALL } \\
\text { 19 Studies } \\
(3,254 / 5,827)\end{array}$ & $\begin{array}{c}\text { AML } \\
\text { 2 Studies } \\
\text { (82/300) }\end{array}$ & $\begin{array}{c}\text { CML } \\
\text { 8 Studies } \\
(1,342 / 1,812)\end{array}$ & $\begin{array}{c}\text { Mixed } \\
5 \text { Studies } \\
(1,226 / 2,884)\end{array}$ \\
\hline $\begin{array}{l}\text { Overall } \\
\text { (62 Studies) }\end{array}$ & NS & $\begin{array}{c}\text { Risk Type: } \\
\text { CC } \\
\text { Protective: } \\
\text { TT + CT }\end{array}$ & NS & NS & NS & NS & NS & NS & NS \\
\hline \multicolumn{10}{|l|}{ Subgroups } \\
\hline $\begin{array}{l}\text { European } \\
\text { (16 Studies) }\end{array}$ & $\begin{array}{c}16 \text { Studies } \\
(3,901 / 5,767) \\
\text { Risk Type: } \\
\text { CC } \\
\text { Protective Type: } \\
\text { TT \& TT+CT }\end{array}$ & $\begin{array}{c}11 \text { Studies } \\
(2,660 / 4,347) \\
\text { Risk Type: } \\
\text { CC } \\
\text { Protective: } \\
\text { CT \& CT+TT }\end{array}$ & $\begin{array}{c}9 \text { Studies } \\
(1,560 / 3,387) \\
\text { NS }\end{array}$ & $\begin{array}{c}2 \text { Studies } \\
(1,100 / 960) \\
\text { NS }\end{array}$ & $\begin{array}{c}5 \text { Studies } \\
(1,223 / 1,420) \\
\text { NS }\end{array}$ & $\begin{array}{c}3 \text { Studies } \\
(357 / 481) \\
\text { NS }\end{array}$ & $\begin{array}{l}0 \\
--\end{array}$ & $\begin{array}{c}2 \text { Studies } \\
\text { (884/939) } \\
\text { NS }\end{array}$ & $\begin{array}{l}0 \\
--\end{array}$ \\
\hline $\begin{array}{l}\text { East Asian } \\
\text { (19 Studies) }\end{array}$ & $\begin{array}{c}19 \text { Studies } \\
(1,242 / 2,872) \\
\text { NS }\end{array}$ & $\begin{array}{c}11 \text { Studies } \\
(1,365 / 1,928) \\
\text { Protective: } \\
\text { CT }\end{array}$ & $\begin{array}{c}10 \text { Studies } \\
(1,198 / 1,688) \\
\text { NS }\end{array}$ & $\begin{array}{c}\text { 1 Study } \\
(87 / 120) \\
\text { NS }\end{array}$ & $\begin{array}{c}8 \text { Studies } \\
(938 / 3,590) \\
\text { NS }\end{array}$ & $\begin{array}{c}\text { 4 Studies } \\
(421 / 1,056) \\
\text { NS }\end{array}$ & $\begin{array}{c}1 \text { Study } \\
(55 / 200) \\
\text { NS }\end{array}$ & $\begin{array}{c}1 \text { Study } \\
(40 / 200) \\
\text { NS }\end{array}$ & $\begin{array}{c}2 \text { Studies } \\
(422 / 2,134) \\
\text { NS }\end{array}$ \\
\hline $\begin{array}{l}\text { South Asian } \\
\text { (9 Studies) }\end{array}$ & $\begin{array}{c}9 \text { Studies } \\
(1,993 / 2,022) \\
\text { NS }\end{array}$ & $\begin{array}{c}8 \text { Studies } \\
(1,576 / 1,530) \\
\text { NS }\end{array}$ & $\begin{array}{c}7 \text { Studies } \\
(1,073 / 1,444) \\
\text { NS }\end{array}$ & $\begin{array}{l}0 \\
--\end{array}$ & $\begin{array}{c}1 \text { Study } \\
(528 / 510) \\
\text { NS }\end{array}$ & $\begin{array}{l}0 \\
--\end{array}$ & $\begin{array}{l}0 \\
--\end{array}$ & $\begin{array}{l}0 \\
--\end{array}$ & $\begin{array}{c}\text { 1 Study } \\
(417 / 492) \\
\text { NS }\end{array}$ \\
\hline $\begin{array}{l}\text { American } \\
\text { (9 Studies) }\end{array}$ & $\begin{array}{c}9 \text { Studies } \\
\text { (848/1,266) } \\
\text { NS }\end{array}$ & $\begin{array}{c}5 \text { Studies } \\
\text { (649/793) } \\
\text { Risk Type: } \\
\text { CC } \\
\text { Protective: } \\
\text { TT \& TT+CT }\end{array}$ & $\begin{array}{c}4 \text { Studies } \\
\text { (505/569) } \\
\text { Risk Type: } \\
\text { CC+CT } \\
\text { Protective: } \\
\text { TT }\end{array}$ & $\begin{array}{c}1 \text { Study } \\
(144 / 224) \\
\text { NS }\end{array}$ & $\begin{array}{c}4 \text { Studies } \\
\text { (199/473) } \\
\text { NS }\end{array}$ & $\begin{array}{l}0 \\
--\end{array}$ & $\begin{array}{c}\text { 1 Study } \\
(27 / 100) \\
\text { NS }\end{array}$ & $\begin{array}{c}3 \text { Studies } \\
(172 / 373) \\
\text { NS }\end{array}$ & $\begin{array}{l}0 \\
--\end{array}$ \\
\hline $\begin{array}{l}\text { Middle East } \\
\text { (7 Studies) }\end{array}$ & $\begin{array}{c}7 \text { Studies } \\
\text { (803/821) } \\
\text { NS }\end{array}$ & $\begin{array}{c}3 \text { Studies } \\
(234 / 311) \\
\text { NS }\end{array}$ & $\begin{array}{c}3 \text { Studies } \\
(234 / 311) \\
\text { NS }\end{array}$ & $\begin{array}{l}0 \\
--\end{array}$ & $\begin{array}{l}4 \text { Studies } \\
(569 / 510) \\
\text { NS }\end{array}$ & $\begin{array}{c}1 \text { Study } \\
(33 / 82) \\
\text { NS }\end{array}$ & $\begin{array}{l}0 \\
---\end{array}$ & $\begin{array}{c}1 \text { Study } \\
(378 / 258) \\
\text { NS }\end{array}$ & $\begin{array}{c}2 \text { Studies } \\
(387 / 258) \\
\text { NS }\end{array}$ \\
\hline $\begin{array}{l}\text { African } \\
\text { (2 Studies) }\end{array}$ & $\begin{array}{l}2 \text { Studies } \\
(185 / 441) \\
\text { NS }\end{array}$ & $\begin{array}{c}1 \text { Study } \\
\text { (88/311) } \\
\text { NS }\end{array}$ & $\begin{array}{c}1 \text { Study } \\
(88,311) \\
\text { NS }\end{array}$ & $\begin{array}{l}0 \\
--\end{array}$ & $\begin{array}{c}1 \text { Study } \\
(97 / 130) \\
\text { NS }\end{array}$ & $\begin{array}{l}0 \\
--\end{array}$ & $\begin{array}{l}0 \\
-\end{array}$ & $\begin{array}{c}1 \text { Study } \\
\text { (97/130) } \\
\text { NS }\end{array}$ & 0 \\
\hline
\end{tabular}

Note:

NS: No Statistical Significance; -- No data;

ALL: Acute Lymphoblastic Leukemia; AML: Acute Myeloid Leukemia; CML: Chronic Myeloid Leukemia.

Table 1B: Schema of significant findings across studies on MTHFR 1298 genotypes and risk of leukemia

\begin{tabular}{|c|c|c|c|c|c|c|c|c|c|}
\hline \multirow[b]{2}{*}{$\begin{array}{c}\text { NTHFR } \\
1298\end{array}$} & \multirow{2}{*}{$\begin{array}{c}\text { All } \\
\mathbf{N}=\mathbf{5 0} \text { Studies } \\
\text { (n Case } / \mathbf{n} \\
\text { Control) } \\
(\mathbf{8 , 3 6 8 / 1 4 , 3 0 1 )}\end{array}$} & \multicolumn{3}{|c|}{ Children } & \multicolumn{5}{|c|}{ Adults } \\
\hline & & $\begin{array}{l}\text { All children } \\
\text { 31 Studies } \\
(5,614 / 8,474)\end{array}$ & $\begin{array}{c}\text { ALL } \\
28 \text { Studies } \\
(4,531 / 7,396)\end{array}$ & $\begin{array}{l}\text { ALL+AML } \\
\text { 4 Studies } \\
(1,114 / 1,326)\end{array}$ & $\begin{array}{c}\text { All adults } \\
\text { 19 Studies } \\
(3,157 / 5,697)\end{array}$ & $\begin{array}{c}\text { ALL } \\
\text { 7 Studies } \\
(767 / 1,537)\end{array}$ & $\begin{array}{c}\text { AML } \\
\text { 2 Studies } \\
\text { (88/300) }\end{array}$ & $\begin{array}{c}\text { CML } \\
\text { 7 Studies } \\
(1,193 / 1,629)\end{array}$ & $\begin{array}{c}\text { Mixed } \\
\text { 3 Studies } \\
(1,151 / 2,231)\end{array}$ \\
\hline $\begin{array}{l}\text { Overall } \\
\text { (50 Studies) }\end{array}$ & NS & NS & NS & NS & NS & NS & NS & $\begin{array}{c}\text { Risk Type: } \\
\text { AA } \\
\text { Protective } \\
\text { CC }=\text { AC }\end{array}$ & NS \\
\hline \multicolumn{10}{|l|}{ Subgroups } \\
\hline $\begin{array}{l}\text { European } \\
\text { (12 Studies) }\end{array}$ & $\begin{array}{c}12 \text { Studies } \\
(3,538 / 4,591) \\
\text { NS }\end{array}$ & $\begin{array}{c}\text { 8 Studies } \\
(2,349 / 3,548) \\
\text { NS }\end{array}$ & $\begin{array}{c}6 \text { Studies } \\
(1,353 / 2,626) \\
\text { NS }\end{array}$ & $\begin{array}{c}2 \text { Studies } \\
\text { (996/958) } \\
\text { Risk Type: } \\
\text { AA } \\
\text { Protective: } \\
\text { CC+AC }\end{array}$ & $\begin{array}{c}4 \text { Studies } \\
(1,189 / 1,367) \\
\text { NS }\end{array}$ & $\begin{array}{c}3 \text { Studies } \\
(357 / 481) \\
\text { NS }\end{array}$ & $\begin{array}{l}0 \\
--\end{array}$ & $\begin{array}{c}1 \text { Study } \\
\text { (836/886) } \\
\text { NS }\end{array}$ & $\begin{array}{l}0 \\
--\end{array}$ \\
\hline $\begin{array}{l}\text { East Asian } \\
\text { (16 Studies) }\end{array}$ & $\begin{array}{c}16 \text { Studies } \\
(2,670 / 5,318) \\
\text { NS }\end{array}$ & $\begin{array}{c}8 \text { Studies } \\
(1,194 / 1,728) \\
\text { NS }\end{array}$ & $\begin{array}{c}7 \text { Studies } \\
(1,107 / 1,608) \\
\text { NS }\end{array}$ & $\begin{array}{l}1 \text { Studies } \\
(87 / 120) \\
\text { NS }\end{array}$ & $\begin{array}{c}8 \text { Studies } \\
(1,476 / 3,590) \\
\text { NS }\end{array}$ & $\begin{array}{c}\text { 4 Studies } \\
(410 / 1056) \\
\text { NS }\end{array}$ & $\begin{array}{c}1 \text { Study } \\
55 / 200 \\
\text { NS }\end{array}$ & $\begin{array}{c}1 \text { Study } \\
(40 / 200) \\
\text { NS }\end{array}$ & $\begin{array}{c}2 \text { Studies } \\
(971 / 2,134) \\
\text { NS }\end{array}$ \\
\hline $\begin{array}{l}\text { South Asian } \\
\text { (6 Studies) }\end{array}$ & $\begin{array}{c}6 \text { Studies } \\
(1,210 / 1,723) \\
\text { NS }\end{array}$ & $\begin{array}{c}6 \text { Studies } \\
(1,210 / 1,723) \\
\text { NS }\end{array}$ & $\begin{array}{c}6 \text { Studies } \\
(1,210 / 1,723) \\
\text { NS }\end{array}$ & 0 & 0 & 0 & 0 & 0 & 0 \\
\hline
\end{tabular}




\begin{tabular}{|c|c|c|c|c|c|c|c|c|c|}
\hline $\begin{array}{l}\text { American } \\
\text { (9 Studies) }\end{array}$ & $\begin{array}{c}9 \text { Studies } \\
(739 / 1,291) \\
\text { NS }\end{array}$ & $\begin{array}{c}5 \text { Studies } \\
(677 / 769) \\
\text { NS }\end{array}$ & $\begin{array}{c}\text { 4 Studies } \\
(606 / 698) \\
\text { NS }\end{array}$ & $\begin{array}{l}\text { 1 Studies } \\
\text { (71/71) } \\
\text { NS }\end{array}$ & $\begin{array}{c}\text { 4 Studies } \\
(199 / 473) \\
\text { NS }\end{array}$ & 0 & $\begin{array}{c}1 \text { Study } \\
27 / 100 \\
\text { NS }\end{array}$ & $\begin{array}{c}3 \text { Studies } \\
\text { (172/373) } \\
\text { Risk Type: } \\
\text { AA } \\
\text { Protective: } \\
\text { CC+AC }\end{array}$ & $\begin{array}{l}0 \\
--\end{array}$ \\
\hline $\begin{array}{l}\text { Mideast } \\
\text { (5 Studies) }\end{array}$ & $\begin{array}{l}5 \text { Studies } \\
(526 / 578)\end{array}$ & $\begin{array}{l}3 \text { Studies } \\
(233 / 311)\end{array}$ & $\begin{array}{l}3 \text { Studies } \\
(233 / 311)\end{array}$ & 0 & $\begin{array}{l}2 \text { Studies } \\
\text { (293/267) }\end{array}$ & 0 & 0 & $\begin{array}{l}\text { 1 Study } \\
(149 / 170)\end{array}$ & $\begin{array}{l}\text { 1 Study } \\
(144 / 97)\end{array}$ \\
\hline & NS & NS & NS & -- & NS & -- & -- & NS & NS \\
\hline $\begin{array}{l}\text { African } \\
\text { (2 Studies) }\end{array}$ & $\begin{array}{c}2 \text { Studies } \\
\text { (185/440) } \\
\text { Risk Type: } \\
\text { AA } \\
\text { Protective: } \\
\text { AC, AC+CC }\end{array}$ & $\begin{array}{c}1 \text { Study } \\
(88 / 310) \\
\text { NS }\end{array}$ & $\begin{array}{c}1 \text { Study } \\
(88 / 310) \\
\text { NS }\end{array}$ & $\begin{array}{l}0 \\
--\end{array}$ & $\begin{array}{c}1 \text { Study } \\
(97 / 130) \\
\text { NS }\end{array}$ & 0 & $\begin{array}{l}0 \\
--\end{array}$ & $\begin{array}{c}1 \text { Study } \\
(97 / 310) \\
\text { NS }\end{array}$ & -- \\
\hline
\end{tabular}

Note:

NS: No Statistical Significance; -- No data;

ALL: Acute Lymphoblastic Leukemia; AML: Acute Myeloid Leukemia; CML: Chronic Myeloid Leukemia.

\section{Table 2: Pooled meta-analysis: MTHFR 677 genotypes and risks of leukemia in children}

\begin{tabular}{|c|c|c|c|c|c|c|c|c|}
\hline \multirow{2}{*}{$\begin{array}{c}\text { Genotype by } \\
\text { Race or Ethnicity } \\
\text { (Number of } \\
\text { studies) }\end{array}$} & \multirow{2}{*}{$\begin{array}{l}\text { Leukemia Case } \\
\qquad \begin{array}{c}(N=6572) \\
n(\%)\end{array}\end{array}$} & \multirow{2}{*}{$\begin{array}{c}\text { Control } \\
(N=9220) \\
n(\%)\end{array}$} & \multicolumn{3}{|c|}{ Test of Heterogeneity } & \multirow{2}{*}{$\begin{array}{c}\text { Statistical } \\
\text { Model }\end{array}$} & \multicolumn{2}{|c|}{ Test of Association } \\
\hline & & & $\mathbf{Q}$ & $p$ & $\mathbf{I}^{2}$ & & $\begin{array}{c}\text { Risk Ratio } \\
(95 \% \mathrm{Cl})\end{array}$ & $p$ \\
\hline TT (39) & $723(11.01)$ & $1064(11.54)$ & 50.00 & 0.0921 & $24 \%$ & Fixed & $0.95(0.87$ to 1.04$)$ & 0.2902 \\
\hline Europe (11) & $284(10.68)$ & $509(11.71)$ & 9.13 & 0.6094 & $0 \%$ & Fixed & $0.91(0.79$ to 1.05$)$ & 0.1938 \\
\hline America (5) & $59(9.09)$ & $100(12.61)$ & 2.301 & 0.6804 & $0 \%$ & Fixed & $0.70(0.52$ to 0.95$)$ & 0.0212 \\
\hline Middle East (3) & $20(8.55)$ & $19(6.11)$ & 1.361 & 0.5064 & $0 \%$ & Fixed & $1.40(0.76$ to 2.59$)$ & 0.2800 \\
\hline South Asia (8) & $86(5.46)$ & $66(4.31)$ & 2.757 & 0.8387 & $0 \%$ & Fixed & $0.90(0.63$ to 1.27$)$ & 0.5411 \\
\hline East Asia (11) & $267(19.56)$ & $350(25.64)$ & 25.88 & 0.0039 & $61.4 \%$ & Random & $1.14(0.87$ to 1.50$)$ & 0.3306 \\
\hline Africa (1) & $7(7.95)$ & $20(6.43)$ & & & & & 1.24 & \\
\hline CT (39) & $2688(40.91)$ & $3921(42.53)$ & 76.89 & 0.0003 & $49.3 \%$ & Random & $0.95(0.90$ to 1.01$)$ & 0.1219 \\
\hline Europe (11) & $1119(42.07)$ & $1953(44.93)$ & 16.93 & 0.1101 & $35 \%$ & Fixed & $0.94(0.89$ to 0.99$)$ & 0.0316 \\
\hline America (5) & $264(40.68)$ & $354(44.64)$ & 8.791 & 0.0665 & $54.5 \%$ & Fixed & $0.91(0.81$ to 1.03$)$ & 0.1434 \\
\hline Middle East (3) & $100(42.74)$ & $126(40.51)$ & 4.185 & 0.1234 & $52.2 \%$ & Fixed & $1.05(0.86$ to 1.28$)$ & 0.6289 \\
\hline South Asia (8) & $568(36.04)$ & $447(29.22)$ & 21.73 & 0.0028 & $67.8 \%$ & Random & $1.12(0.90$ to 1.38$)$ & 0.3090 \\
\hline East Asia (11) & $595(43.59)$ & $906(66.37)$ & 18.26 & 0.0507 & $45.2 \%$ & Fixed & $0.93(0.86$ to 1.00$)$ & 0.0491 \\
\hline Africa (1) & $42(47.73)$ & $135(43.41)$ & & & & & 1.10 & \\
\hline CC (39) & $3161(48.10)$ & $4235(45.93)$ & 95.06 & $<0.0001$ & $59 \%$ & Random & $1.04(1.01$ to 1.08$)$ & 0.0139 \\
\hline Europe (11) & $1257(47.26)$ & $1885(43.26)$ & 22.85 & 0.0113 & $56.2 \%$ & Random & $1.11(1.02$ to 1.22$)$ & 0.0207 \\
\hline America (5) & $326(50.23)$ & $339(42.75)$ & 10.33 & 0.0352 & $61.3 \%$ & Random & $1.09(1.02$ to 1.20$)$ & 0.0451 \\
\hline Middle East (3) & $114(48.72)$ & $166(53.38)$ & 3.135 & 0.2086 & $36.2 \%$ & Fixed & $0.92(0.77$ to 1.08$)$ & 0.3047 \\
\hline South Asia (8) & $922(58.50)$ & $1017(66.47)$ & 25.76 & 0.0006 & $72.8 \%$ & Random & $0.95(0.86$ to 1.06$)$ & 0.3603 \\
\hline East Asia (11) & $503(36.85)$ & $672(49.23)$ & 12.59 & 0.2476 & $20.6 \%$ & Fixed & 1.07 (0.97 to 1.17$)$ & 0.1735 \\
\hline Africa (1) & $39(44.32)$ & $156(50.16)$ & & & & & 0.88 & \\
\hline TT + CT (39) & $3411(51.90)$ & $4985(54.07)$ & 81.99 & $<0.0001$ & $53.7 \%$ & Random & $0.96(0.93$ to 0.99$)$ & 0.0154 \\
\hline Europe (11) & $1403(52.74)$ & $2462(56.64)$ & 18.89 & 0.0417 & $47.1 \%$ & Random & 0.94 (0.90to 0.99$)$ & 0.0187 \\
\hline America (5) & $323(49.77)$ & $454(57.25)$ & 12.35 & 0.0149 & $67.6 \%$ & Random & 0.83 (0.68 to 0.99$)$ & 0.0449 \\
\hline Middle East (3) & $120(51.28)$ & $145(46.62)$ & 3.36 & 0.1867 & $40.4 \%$ & Fixed & $1.09(0.92$ to 1.30$)$ & 0.3 \\
\hline South Asia (8) & $654(41.49)$ & $513(33.53)$ & 25.60 & 0.0006 & $72.7 \%$ & Random & $1.10(0.89$ to 1.36$)$ & 0.3572 \\
\hline East Asia (11) & $862(63.15)$ & $1256(65.15)$ & 11.60 & 0.3126 & $13.8 \%$ & Fixed & $0.97(0.92$ to 1.02$)$ & 0.1761 \\
\hline Africa (1) & $49(55.68)$ & $155(49.84)$ & & & & & 1.12 & \\
\hline $\mathrm{CC}+\mathrm{CT}$ (39) & 5849 (88.99) & $8156(88.46)$ & 6.50 & $>0.9999$ & $0 \%$ & Fixed & $1.34(0.85$ to 2.10$)$ & 0.2039 \\
\hline
\end{tabular}




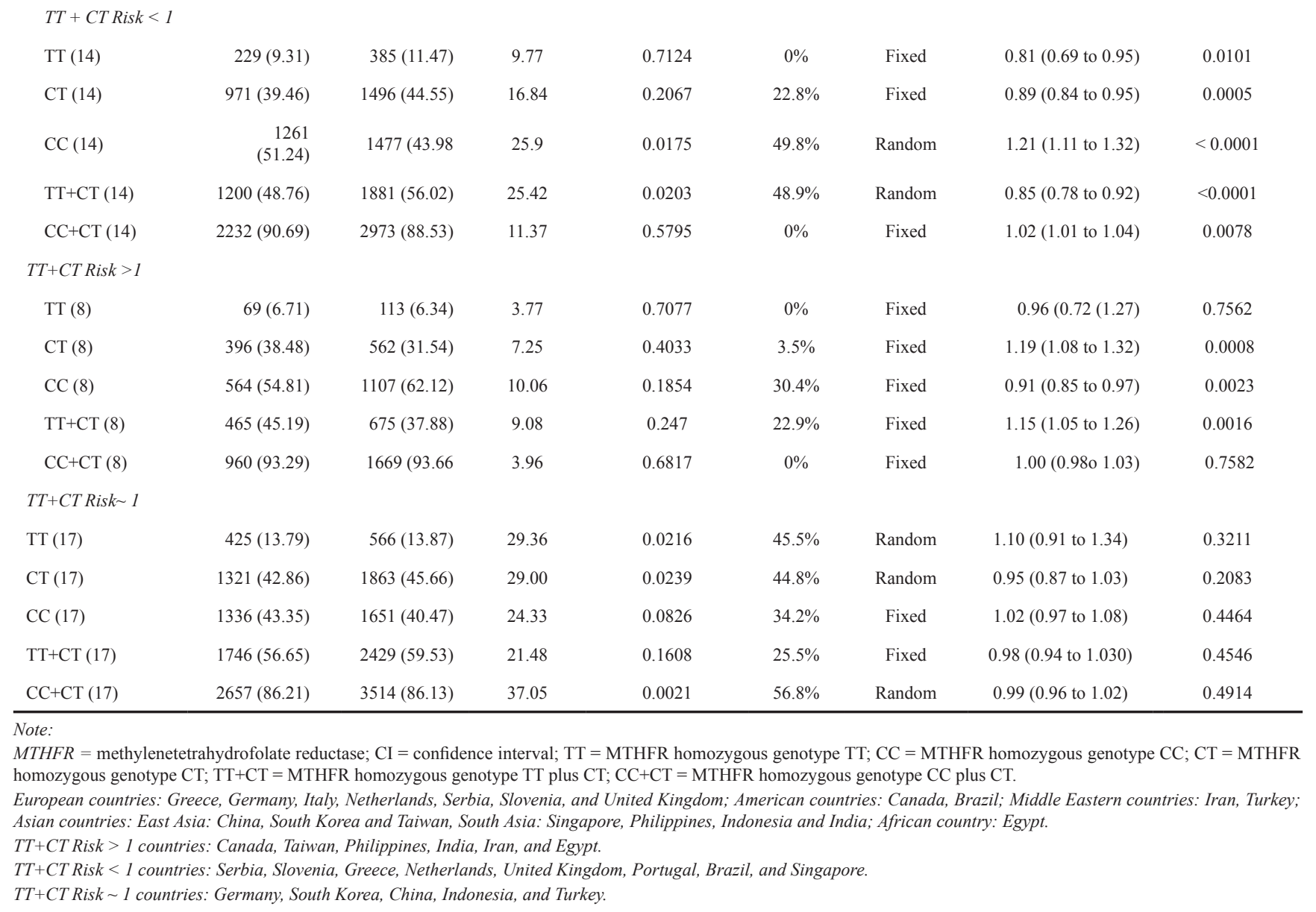

high air pollution on the percentage of MTHFR 677 TT polymorphism in childhood leukemias (mean difference $=+8.7 \%, 95 \%$ CI $[9 \%, 16.6 \%], p=.0258)$. To further illustrate this significance, we plotted those results on nonlinear curves. With the increased air pollution rates from low (Level 2) to high (Level 4), there was a steeper increase in the percentages of MTHFR 677 TT polymorphisms in the leukemia-case groups than in control groups (Supplementary Figure S5). The heat map also demonstrated dense distribution of the MTHFR 677 TT mutation in leukemia-case groups (more red blocks) than in control groups (Supplementary Figure S6).

\section{DISCUSSION}

To date, many studies have investigated the association between MTHFR polymorphisms and leukemias; however, results have been inconsistent. Consistent with previous analyses [4, 12, 27, 34-36], our comprehensive analysis generated from studies to date indicated significant heterogeneity in the distributions of MTHFR polymorphisms across age groups, regions, and types of leukemia. We found MTHFR 677 TT and CT polymorphisms played protective roles, while the CC wildtype was a risk genotype for leukemia in all child populations worldwide combined, as well as in children from Europe, American, and East Asia. In addition, MTHFR 1298 polymorphisms were protective against ALL and AML in children from Europe, as well as CML in adults. Our analyses by countries in subgroups showed that MTHFR 677 polymorphisms were protective mainly in European countries but leukemogenic in South Asian and African countries. Such variations among world populations might be associated with different underpinning mechanisms and pathways for the effects of MTHFR 677 polymorphisms in childhood leukemia in relation to geographic locations $[5,7,8]$.

Furthermore, using meta-predictive techniques, we showed a potential impact of air pollution on the link between MTHFR gene polymorphisms and leukemogenesis. In countries with high levels of air pollution (level 4), MTHFR 677 polymorphisms played a protective role in European countries (i.e., UK, Greece, Portugal), had mixed effects in China and Turkey, and posed a risk for childhood leukemia in Taiwan and Iran. Among countries with low levels of air pollution (level 2), MTHFR 677 polymorphisms played protective roles against childhood leukemia in Brazil but were leukemogenic in India. These findings illustrate the complexity of gene-environment interactions across regions and ethnic groups. Air pollution has direct $[18,19]$ and indirect effects $[13,17,24]$ on the 
Table 3: Meta-prediction: Air pollution associated death (AP Death) on MTHFR 677 genotypes for controls (ct) and leukemia cases (ca) in children

\begin{tabular}{|c|c|c|c|c|c|c|c|c|c|c|c|}
\hline \multirow[b]{2}{*}{ Variable } & \multirow[b]{2}{*}{$\mathrm{AICc}$} & \multicolumn{4}{|c|}{ Partition tree } & \multicolumn{6}{|c|}{ Tukey's test } \\
\hline & & $\begin{array}{c}\text { AP } \\
\text { Death }\end{array}$ & Count & Mean & SD & $\begin{array}{c}\text { Level } \\
\text { Compared }\end{array}$ & Difference & $\begin{array}{c}\text { SE } \\
\text { Difference }\end{array}$ & $\begin{array}{c}\text { Lower } \\
\text { CI }\end{array}$ & $\begin{array}{c}\text { Upper } \\
\text { CI }\end{array}$ & $p$ \\
\hline \multirow[t]{3}{*}{ TT\%ct } & -100.35 & $2 \& 3$ & 21 & 0.096 & 0.063 & $4 / 2$ & 0.045 & 0.028 & -0.024 & 0.115 & 0.2605 \\
\hline & & 4 & 18 & 0.126 & 0.063 & $4 / 3$ & 0.026 & 0.023 & -0.083 & 0.083 & 0.5104 \\
\hline & & & & & & $3 / 2$ & 0.019 & 0.029 & -0.054 & 0.093 & 0.7936 \\
\hline \multirow[t]{3}{*}{ ТT\%са } & -91.168 & $2 \& 3$ & 21 & 0.076 & 0.045 & $4 / 2$ & 0.087 & 0.032 & 0.009 & 0.166 & 0.0258 \\
\hline & & 4 & 18 & 0.143 & 0.092 & $4 / 3$ & 0.054 & 0.026 & -0.010 & 0.118 & 0.1094 \\
\hline & & & & & & $3 / 2$ & 0.033 & 0.034 & -0.049 & 0.116 & 0.5910 \\
\hline \multirow[t]{3}{*}{$\mathrm{CT} \% \mathrm{ct}$} & -63.886 & $2 \& 3$ & 21 & 0.389 & 0.113 & $4 / 2$ & 0.074 & 0.046 & -0.037 & 0.186 & 0.2476 \\
\hline & & 4 & 18 & 0.452 & 0.083 & $4 / 3$ & 0.063 & 0.037 & -0.029 & 0.154 & 0.2262 \\
\hline & & & & & & $3 / 2$ & 0.012 & 0.048 & -0.106 & 0.129 & 0.9686 \\
\hline \multirow[t]{3}{*}{ СТ\%ca } & -63.295 & 2 & 8 & 0.365 & 0.121 & $3 / 2$ & 0.040 & 0.049 & -0.080 & 0.159 & 0.6995 \\
\hline & & $3 \& 4$ & 31 & 0.401 & 0.096 & $4 / 2$ & 0.031 & 0.046 & -0.083 & 0.144 & 0.7842 \\
\hline & & & & & & $3 / 4$ & 0.009 & 0.038 & -0.084 & 0.101 & 0.9719 \\
\hline \multirow[t]{3}{*}{$\mathrm{CC} \% \mathrm{ct}$} & -35.288 & 4 & 18 & 0.422 & 0.115 & $2 / 4$ & 0.120 & 0.066 & -0.041 & 0.280 & 0.1762 \\
\hline & & $2 \& 3$ & 21 & 0.515 & 0.166 & $3 / 4$ & 0.089 & 0.054 & -0.042 & 0.220 & 0.2367 \\
\hline & & & & & & $2 / 3$ & 0.031 & 0.069 & -0.138 & 0.200 & 0.8950 \\
\hline \multirow[t]{3}{*}{$\mathrm{CC} \%$ ca } & -41.963 & $3 \& 4$ & 31 & 0.479 & 0.132 & $2 / 4$ & 0.118 & 0.060 & -0.029 & 0.266 & 0.1360 \\
\hline & & 2 & 8 & 0.578 & 0.138 & $2 / 3$ & 0.073 & 0.063 & -0.082 & 0.228 & 0.4926 \\
\hline & & & & & & $3 / 4$ & 0.046 & 0.049 & -0.075 & 0.166 & 0.6265 \\
\hline \multirow[t]{3}{*}{ RRTT } & 66.809 & $2 \& 3$ & 19 & 0.832 & 0.286 & $4 / 2$ & 0.399 & 0.270 & -0.263 & 1.062 & 0.3134 \\
\hline & & 4 & 18 & 1.224 & 0.748 & $4 / 3$ & 0.357 & 0.213 & -0.167 & 0.881 & 0.2312 \\
\hline & & & & & & $3 / 2$ & 0.043 & 0.286 & -0.659 & 0.745 & 0.9878 \\
\hline \multirow[t]{3}{*}{ RRCT } & 1.202 & 4 & 18 & 0.904 & 0.241 & $3 / 4$ & 0.146 & 0.085 & -0.062 & 0.354 & 0.2129 \\
\hline & & $2 \& 3$ & 21 & 1.025 & 0.223 & $2 / 4$ & 0.107 & 0.104 & -0.148 & 0.362 & 0.5655 \\
\hline & & & & & & $3 / 2$ & 0.040 & 0.110 & -0.229 & 0.308 & 0.9317 \\
\hline \multirow[t]{3}{*}{ RRCC } & 14.865 & $3 \& 4$ & 31 & 1.079 & 0.257 & $4 / 3$ & 0.139 & 0.099 & -0.102 & 0.381 & 0.3466 \\
\hline & & 2 & 8 & 1.176 & 0.345 & $2 / 3$ & 0.137 & 0.127 & -0.174 & 0.449 & 0.5339 \\
\hline & & & & & & $4 / 2$ & 0.002 & 0.121 & -0.294 & 0.298 & 0.9998 \\
\hline
\end{tabular}

Note. MTHFR = methylenetetrahydrofolate reductase $\mathrm{CI}=$ confidence interval; $\mathrm{AICc}=$ Akaike's information criterion correction; $\mathrm{AP}$ Death $=$ annual death rate from air pollution, in levels per million people $(2=50-100$ deaths, $3=100-250$ deaths, $4=250-400+$ deaths $)$; RR = Risk Ratio; $\mathrm{ct}=$ controls; $\mathrm{ca}=$ Leukemia cases; TT\%ct $=$ the percentages of TT mutations in the control group; TT $\% c a=$ the percentages of TT mutations in the Leukemia cases; CT\%ct = the percentages of CT mutations in the control group; CT $\%$ ca $=$ the percentages of CT mutations in the Leukemia cases; $\mathrm{CC} \% \mathrm{ct}=$ the percentages of $\mathrm{CC}$ genotype in the control group; $\mathrm{CC} \% \mathrm{ca}=$ the percentages of $\mathrm{CC}$ genotype in the Leukemia cases; RRTT = risk ratio of TT mutation between case and control groups; RRCT = risk ratio of CT mutation between case and control groups; $\mathrm{RRCC}=$ risk ratio of $\mathrm{CC}$ genotype between case and control groups.

development of childhood leukemia, possibly aggravated more in warmer regions. Given that we do not yet have sufficient studies, especially in regions other than Europe and China, further studies are warranted to examine the mechanisms of MTHFR polymorphisms in leukemias. In summary, research should study how genes, environment, and additional risk factors including air pollution interact with MTHFR polymorphisms; such studies across different regions and ethnicities will advance the prevention of leukemia, especially for children. 


\section{Relative risk meta-analysis plot (random effects)}

Serbia Damnjanovic $2010\langle\mathrm{n}=490$ ) Slovenia Petra $2007(n=326)$ Greece Chatzidakis 2006 ( $n=140$ ) Greece Karathansis $2011(n=83)$ Netherland de Jonge $2009\{n=741\rangle$ Netherland te Winkel 2011 ( $\mathrm{n}=230$ ) UK Wiemels 2001 ( $n=416$ ) UK Lightfoot $2010(n=1644)$ Portugal Oliveira $2005\{n=214$ ) Brazil Zanrosso $2006(n=158)$ Brazil France 2001 ( $n=141$ ) Brazil Silvia $2013(\mathrm{n}=368)$ Brazil Zanrosso $2006(n=205)$ Singapore Yeoh 2009 ( $n=663$ ) combined [random] 0.3

\section{Relative risk meta-analysis plot (fixed effects)}

Canada karjinovic 2004 ( $n=570$ )

Taiwan Liu $2008(\mathrm{n}=266)$

Philippines Alcasabas $2008(n=583)$

India Reddy $2006(n=277)$

India Sadananda $2010(n=185)$

India Sood $2010(\mathrm{n}=350)$

Iran Azhar $2012(n=181)$

Egypt Kamel 2007 ( $n=399)$

combined [fixed]

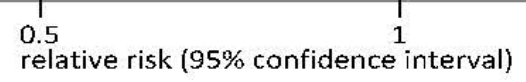

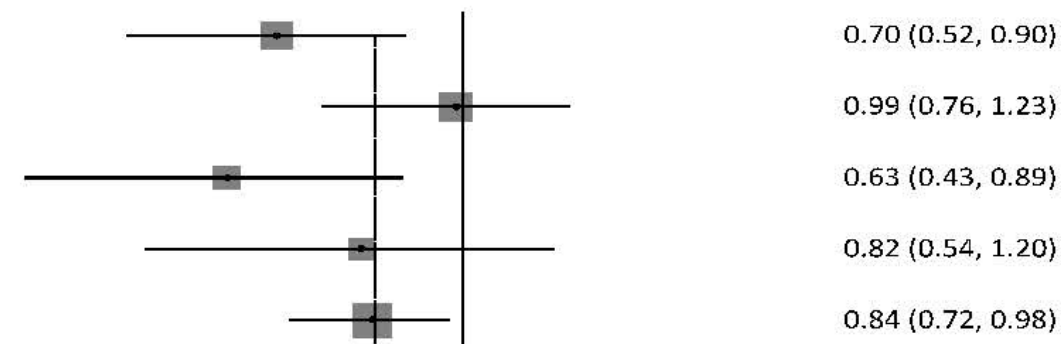

$0.85(0.65,1.10)$

$0.98(0.83,1.17)$

$0.99(0.91,1.09)$

$0.90(0.70,1.14)$

$0.62(0.42,0.89)$

$0.70(0.52,0.93)$

$0.75(0.60,0.92)$

$0.99(0.75,1.30)$

$0.80(0.68,0.94)$

$0.85(0.78,0.92)$

$1.01(0.88,1.16)$

$1.01(0.74,1.34)$

$1.27(0.91,1.77)$

$1.40(1.12,1.77)$

$1.07(0.54,2.12)$

$1.34(0.99,1.78)$

$1.27(0.92,1.75)$

$1.12(0.89,1.37)$

$1.15(1.05,1.26)$ 


\section{Relative risk meta-analysis plot (fixed effects)}

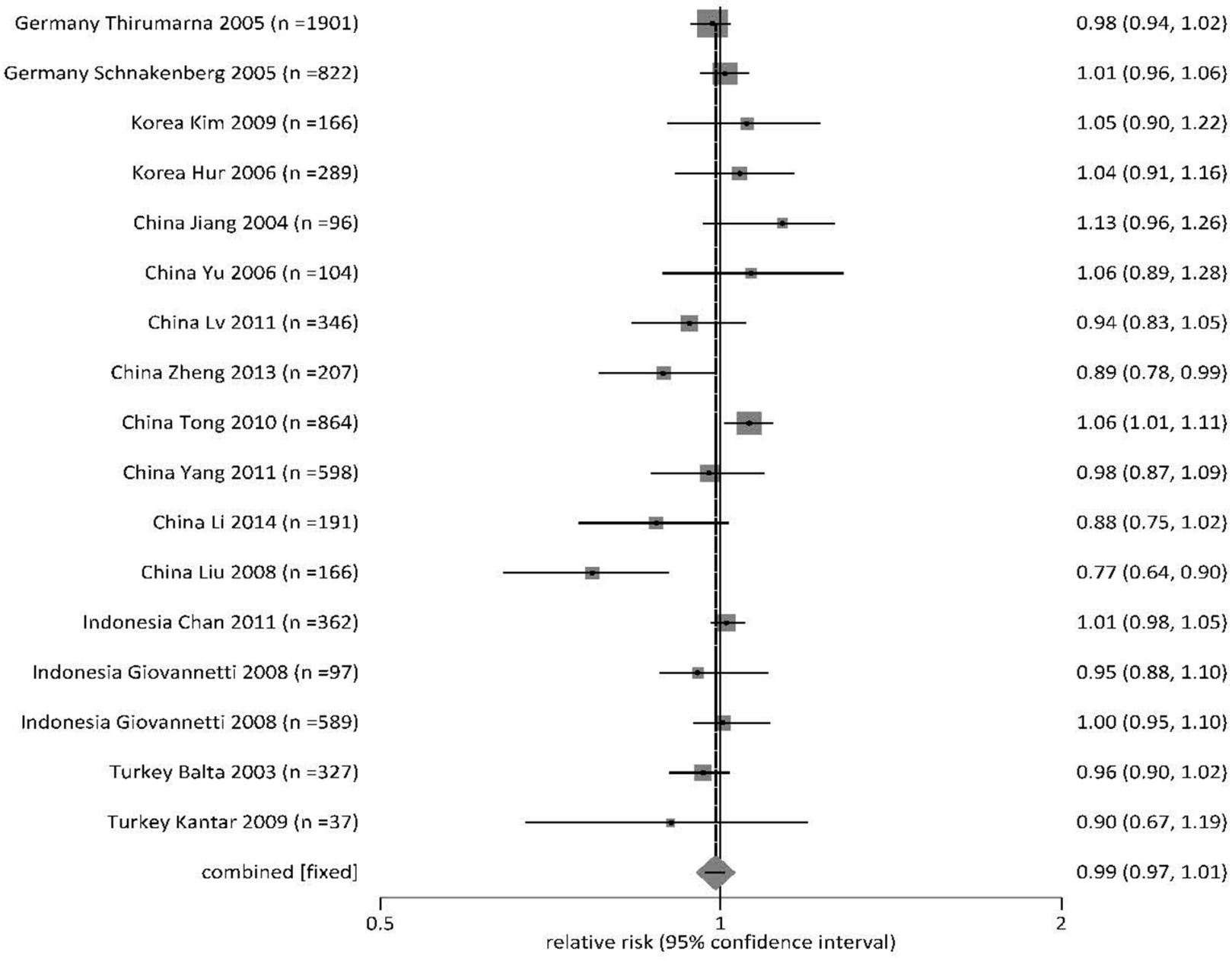

Figure 2: Forest plot for meta-analysis of MTHFR 677 TT and CT polymorphisms on risk of leukemia in children.

\section{MATERIALS AND METHODS}

\section{Study search strategy}

We followed the guidelines for Preferred Reporting Items of Systematic Reviews and Meta-Analysis (PRISMA) [37], and conducted a comprehensive literature search from databases of MEDLINE (PubMed), Cochrane Library, Embase, e-Books, EBSCO, and PsychoInfo from 1965 to August, 2016. The search strategy included the following keywords and terms: "MTHFR gene" or "leukemia" and "adult" or "children," "environment," "diet prevention," "folate," "lifestyle," "behavior," "case control design" and "meta-analysis," and all leukemia types. Additionally, previous meta-analysis and review papers were used to cross check and trace back all original studies. Various databases were searched repeatedly at three different times at least 3 months apart until no additional eligible papers were identified.

\section{Inclusion/exclusion criteria}

The inclusion criteria were that the articles 1) examined the association of the MTHFR polymorphisms (C677T and/ or A1298C) and leukemia risk using a case-control design, 2 ) included the genotype frequency in both case and control groups, 3) were written in English or 4) were written in nonEnglish but provided tables with genotype allele frequencies for both case and control groups. Articles were excluded if they 1) were written in non-English without tables listing genotype allele counts, 2) were not case-control studies, or 3) had missing counts of genotype frequency.

Based on those criteria, we first identified 92 articles. We excluded 38 articles, including 11 metaanalyses, 7 non-case-control studies, 8 studies on other neoplasms or carcinomas, and 12 studies that lacked genotype allele counts or duplicates. The remaining 54 papers were included in the final analyses 
(Figure 1). Two raters independently conducted the process of literature search, screening, data collection, and extraction based on the study protocol. The discrepancy between raters was reduced to zero prior to data analyses.

\section{Quality assessment}

We assessed the quality of these studies [38] based on the criteria from various sources including QUOROM and PRISMA guidelines, and standards for related human genome studies [37, 39]. We assessed domains that included external validity for grouping data (score range of 0-11), internal validity for DNA analysis process and procedural rigor (score range of 0-12), and the quality of detailed reporting on limiting bias (score range of 0-7). Thus, the ranges for each possible total score were from 0 to 30 . All included studies showed quality scores from 15 to 22 ; thus, all 54 studies scored in the top $50 \%$ of our range, so we judged their findings trustworthy [39].

\section{Data synthesis and analysis}

We entered data into Excel, and analyzed data using StatsDirect Version 3 updated software (Cheshire, UK). We calculated pooled risk ratios (RR), odds ratios (OR), and $95 \% \mathrm{CI}$ for the MTHFR subtypes, distinguishing between cases and controls, to test for the associations of genotypes with leukemia. We compared pooled standardized RRs versus ORs to check the differences between the two ratios and found RRs more conservative with less significant findings; therefore, we used RRs in this report.

We used SAS JMP ${ }^{\circledR}$ pro 13 program (SAS Institute, New York, NY, 2016) for meta-prediction analyses to examine how AP death was related to the MTHFR polymorphisms and leukemia risk [40]. In addition to conventional analyses, we used big-data analytics including partition trees for these grouping analyses. The goodness of fit was judged by the Akaike's information criterion correction (AICc), with a smaller AIC suggesting a better model [41-43]. To produce more accurate metaprediction, we used multiple methods to triangulate and cross-validate the findings - including nonlinear fit modeling, partition trees, heat maps, one-way ANOVA, and Tukey's posthoc tests. To compare AICc results with the partition trees, we used Tukey's tests [44]. All $p$ values were two-tailed with a significance level at .05. We used the GIS maps to demonstrate the distributions of mutations and their associations with leukemia risks on the global maps $[30,45]$.

We used Hardy-Weinberg Equilibrium (HWE) analysis to detect the discrepancies in genotype distributions [46, 47]. HWE results were significant in five studies $(p<0.05)$. We performed subgroup analyses on those five studies versus those that had acceptable HWE. However, the results did not yield significant differences.
Therefore, all studies regardless of HWE status were included in the final meta-analysis (Supplementary Table S1). Egger's test and funnel plots were performed to detect publication bias $[48,49]$.

\section{CONFLICTS OF INTEREST}

The authors declared no potential financial conflicts of interest and no industrial support.

\section{REFERENCES}

1. McNeil D, Coté T, Clegg L, Mauer A. SEER update of incidence and trends in pediatric malignancies: acute lymphoblastic leukemia. Med Pediatr Oncol. 2002; 39:554-557.

2. Reaman GH, Smith FO. Childhood leukemia. Springer; 2014.

3. American Cancer Society. Cancer Facts \& Figures 2015. Atlanta: American Cancer Society. 2015.

4. Qin YT, Zhang Y, Wu F, Su Y, Lu GN, Wang RS. Association between MTHFR polymorphisms and acute myeloid leukemia risk: a meta-analysis. PloS one. 2014; 9:e88823.

5. Aly RM, Taalab MM, Ghazy HF. MTHFR A1298C, C677T gene polymorphisms and susceptibility to chronic myeloid leukemia in Egypt. Int J Clin Exp Pathol. 2014; 7:2571.

6. Sazawal S, Chaubey R, Kaur P, Chikkara S, Kumar B, Bakshi S, Arya L, Raina V, Gupta AD, Saxena R. MTHFR gene polymorphisms and the risk of acute lymphoblastic leukemia in adults and children: a case control study in India. Indian J Hematol Blood Transfus. 2014; 30:219-225.

7. Kim HN, Kim YK, Lee IK, Yang DH, Lee JJ, Shin MH, Park KS, Choi JS, Park MR, Jo DY. Association between polymorphisms of folate-metabolizing enzymes and hematological malignancies. Leuk Res. 2009; 33:82-87.

8. Pereira TV, Rudnicki M, Pereira AC, Pombo-deOliveira MS, Franco RF. 5,10-Methylenetetrahydrofolate reductase polymorphisms and acute lymphoblastic leukemia risk: a meta-analysis. Cancer Epidemiol Biomarkers Prev. 2006; 15:1956-1963.

9. Krajinovic M, Lamothe S, Labuda D, LemieuxBlanchard É, Théorêt Y, Moghrabi A, Sinnett D. Role of MTHFR genetic polymorphisms in the susceptibility to childhood acute lymphoblastic leukemia. Blood. 2004; 103:252-257.

10. Kamel Azza M, Heba SM, Gamal TE, Rong RB, GB K. Synergistic effect of methyltetrahydrofolate reductase (MTHFR) C677T, A1298C polymorphism as risk modifiers of pediatric acute lymphoblastic leukemia. J Egypt Natl Canc Inst. 2007; 19:96-105.

11. Hirschey MD, DeBerardinis RJ, Diehl AME, Drew JE, Frezza C, Green MF, Jones LW, Ko YH, Le A, Lea MA. Dysregulated metabolism contributes to oncogenesis. Seminars in cancer biology: Elsevier. 2015; S129-S150. 
12. Yan J, Yin M, Dreyer ZE, Scheurer ME, Kamdar K, Wei Q, Okcu MF. A meta-analysis of MTHFR C677T, A1298C polymorphisms and risk of acute lymphoblastic leukemia in children. Pediatr Blood Cancer. 2012; 58:513-518.

13. Stewart B, Wild CP. World cancer report 2014. International Agency for Research on Cancer, World Health organization. 2014.

14. Franco RF, Simoes BP, Tone LG, Gabellini SM, Zago MA, Falcão RP. The methylenetetrahydrofolate reductase C677T gene polymorphism decreases the risk of childhood acute lymphocytic leukaemia. Br J Haematol. 2001; 115:616-618.

15. Duthie SJ. Folic acid deficiency and cancer: mechanisms of DNA instability. Br Med Bull. 1999; 55:578-592.

16. Wiemels JL, Smith RN, Taylor GM, Eden OB, Alexander FE, Greaves MF and investigators UKCCS. Methylenetetrahydrofolate reductase (MTHFR) polymorphisms and risk of molecularly defined subtypes of childhood acute leukemia. Proceedings of the National Academy of Sciences. 2001; 98:4004-4009.

17. Filippini T, Heck JE, Malagoli C, Giovane CD, Vinceti M. A review and meta-analysis of outdoor air pollution and risk of childhood leukemia. J Environ Sci Health C Environ Carcinog Ecotoxicol Rev. 2015; 33:36-66.

18. Gao Y, Zhang Y, Kamijima M, Sakai K, Khalequzzaman M, Nakajima T, Shi R, Wang X, Chen D, Ji X. Quantitative assessments of indoor air pollution and the risk of childhood acute leukemia in Shanghai. Environ pollut. 2014; 187:81-89.

19. Metayer C, Scelo G, Chokkalingam AP, Barcellos LF, Aldrich MC, Chang JS, Guha N, Urayama KY, Hansen HM, Block G, Kiley V, Wiencke JK, Wiemels JL, et al. Genetic variants in the folate pathway and risk of childhood acute lymphoblastic leukemia. Cancer Causes Control. 2011; 22:1243-1258.

20. Badaloni C, Ranucci A, Cesaroni G, Zanini G, Vienneau D, Al-Aidrous F, De Hoogh K, Magnani C, Forastiere F, Magnani C. Air pollution and childhood leukaemia: a nationwide case-control study in Italy. J Occup Environ Med. 2013:oemed-2013-101604.

21. Amigou A, Sermage-Faure C, Orsi L, Leverger G, Baruchel A, Bertrand Y, Nelken B, Robert A, Michel G, Margueritte G. Road traffic and childhood leukemia: the ESCALE study (SFCE). Environ Health Perspect. 2011; 119:566.

22. Bailey HD, de Klerk NH, Fritschi L, Attia J, Daubenton JD, Armstrong BK, Milne E. Refuelling of vehicles, the use of wood burners and the risk of acute lymphoblastic leukaemia in childhood. Paediatr Perinat Epidemiol. 2011; 25:528-539.

23. Ding G, Shi R, Gao Y, Zhang Y, Kamijima M, Sakai K, Wang G, Feng C, Tian Y. Pyrethroid pesticide exposure and risk of childhood acute lymphocytic leukemia in Shanghai. Environ sci technol. 2012; 46:13480-13487.

24. Buffler PA, Kwan ML, Reynolds P, Urayama KY. Environmental and genetic risk factors for childhood leukemia: appraising the evidence. Cancer Invest. 2005; 23:60-75.
25. Robien K, Ulrich C. 5, 10-Methylenetetrahydrofolate reductase polymorphisms and leukemia risk: a HuGE minireview. Am J Epidemiol. 2003; 157:571-582.

26. Jiang Y, Hou J, Zhang Q, Jia ST, Wang BY, Zhang JH, Tang WR, Luo Y. The MTHFR C677T polymorphism and risk of acute lymphoblastic leukemia: an updated metaanalysis based on 37 case-control studies. Asian Pac J Cancer Prev. 2013; 14:6357-6362.

27. Wang $\mathrm{H}$, Wang J, Zhao L, Liu X, Mi W. Methylenetetrahydrofolate reductase polymorphisms and risk of acute lymphoblastic leukemia-evidence from an updated meta-analysis including 35 studies. BMC Med Genet. 2012; 13:77.

28. Tong N, Sheng X, Wang M, Fang Y, Shi D, Zhang Z, Zhang Z. Methylenetetrahydrofolate reductase gene polymorphisms and acute lymphoblastic leukemia risk: a meta-analysis based on 28 case-control studies. Leuk Lymphoma. 2011; 52:1949-1960.

29. Zintzaras E, Koufakis T, Ziakas PD, Rodopoulou P, Giannouli S, Voulgarelis M. A meta-analysis of genotypes and haplotypes of methylenetetrahydrofolate reductase gene polymorphisms in acute lymphoblastic leukemia. Eur J Epidemiol. 2006; 21:501-510.

30. Quantum G. Development Team, 2012. Quantum GIS geographic information system. Open source geospatial foundation project. Free Software Foundation, India. 2013.

31. Environmental Systems Research Institute. What is GIS? (http://www.esri.com/what-is-gis. 2016.

32. World Health Organization. The Health and Enviorment Linkage Initiavtive: Scientific assessment tools. (http:// www.who.int/heli/tools/en/. 2016.

33. World Health Organization. Global health risks. (https:// commons.wikimedia.org/wiki/File:Deaths_from_air_ pollution.png 2012.

34. Li Sy, Ye Jy, Liang Ey, Zhou Lx, Yang M. Association between MTHFR C677T polymorphism and risk of acute lymphoblastic leukemia: a meta-analysis based on 51 casecontrol studies. Med Sci Monit. 2015; 21:740-748.

35. Wang $\mathrm{H}$, Meng L, Zhao L, Wang J, Liu X, Mi W. Methylenetetrahydrofolate reductase polymorphism C677T is a protective factor for pediatric acute lymphoblastic leukemia in the Chinese population: a meta-analysis. Genet Test Mol Biomarkers. 2012; 16:1401-1407.

36. Koppen IJ, Hermans FJ, Kaspers GJ. Folate related gene polymorphisms and susceptibility to develop childhood acute lymphoblastic leukaemia. Br J Haematol. 2010; 148:3-14.

37. Moher D, Liberati A, Tetzlaff J, Altman D. Preferred reporting items for systematic reviews and meta-analyses: the PRISMA statement. Ann Intern Med. 2009; 151:264-269.

38. Shiao S, Yu C. Meta-Prediction of MTHFR Gene Polymorphism Mutations and Associated Risk for Colorectal Cancer. Biol Res Nurs. 2016:1099800415628054.

39. Moher D, Cook D, Eastwood S, Olkin I, Rennie D, Stroup D. [Improving the quality of reports of meta- 
analyses of randomized controlled trials: the QUOROM Statement]. Rev Esp Salud Publica. 1999; 74:107-118.

40. Speybroeck N. Classification and regression trees. Int $\mathbf{J}$ Public Health Res. 2012; 57:243-246.

41. Bozdogan H. Model selection and Akaike's information criterion (AIC): The general theory and its analytical extensions. Psychometrika. 1987; 52:345-370.

42. Akaike H. Maximum likelihood identification of Gaussian autoregressive moving average models. Biometrika. 1973; 60:255-265.

43. Pan W. Akaike's information criterion in generalized estimating equations. Biometrics. 2001; 57:120-125.

44. Jaccard J, Becker MA, Wood G. Pairwise multiple comparison procedures: a review. Psychol Bull. 1984; 96:589.

45. Clarke KC. Getting started with geographic information systems: Prentice Hall Upper Saddle River, NJ. 1997.
46. Hosking L, Lumsden S, Lewis K, Yeo A, McCarthy L, Bansal A, Riley J, Purvis I, Xu CF. Detection of genotyping errors by Hardy-Weinberg equilibrium testing. Eur J Hum Genet. 2004; 12:395-399.

47. Salanti G, Amountza G, Ntzani E, Ioannidis J. HardyWeinberg equilibrium in genetic association studies: an empirical evaluation of reporting, deviations, and power. Eur J Hum Genet. 2005; 13:840-848.

48. Duval S, Tweedie R. Trim and fill: a simple funnel-plotbased method of testing and adjusting for publication bias in meta-analysis. Biometrics. 2000; 56:455-463.

49. Egger M, Smith GD, Schneider M, Minder C. Bias in metaanalysis detected by a simple, graphical test. BMJ. 1997; 315:629-634. 